\title{
Overview of Recent and Current Research on the TCV Tokamak
}

\author{
S. Coda for the TCV team ${ }^{1}$
}

Ecole Polytechnique Fédérale de Lausanne (EPFL), Centre de Recherches en Physique des Plasmas, Association EURATOM-Confédération Suisse, CH-1015 Lausanne, Switzerland

E-mail contact of main author: stefano.coda@epfl.ch

\begin{abstract}
Through a diverse research program, TCV addresses physics issues and develops tools for ITER and for the longer-term goals of nuclear fusion, relying especially on its extreme plasma shaping and ECRH launching flexibility and preparing for an ECRH and NBI power upgrade. Localized edge heating was unexpectedly found to decrease the period and relative energy loss of ELMs. Successful ELM pacing has been demonstrated by following individual ELM detection with an ECRH power cut before turning the power back up to trigger the next ELM, the duration of the cut determining the ELM frequency. Negative triangularity was also seen to reduce the ELM energy release. H-mode studies have focused on the L-H threshold dependence on the main ion species and on the divertor leg length. Both L- and H-modes have been explored in the snowflake configuration with emphasis on edge measurements, revealing that the heat flux to the strike points on the secondary separatrix increases as the X-points approach each other, well before they coalesce. In L-mode, a systematic scan of the auxiliary power deposition profile, with no effect on confinement, has ruled it out as the cause of confinement degradation. An ECRH power absorption observer based on transmitted stray radiation was validated for eventual polarization control. A new profile control methodology was introduced, relying on real-time modeling to supplement diagnostic information; the RAPTOR current transport code in particular has been employed for joint control of the internal inductance and central temperature. An internal inductance controller using the Ohmic transformer has also been demonstrated. Fundamental investigations of NTM seed island formation by sawtooth crashes and of NTM destabilization in the absence of a sawtooth trigger were carried out. Both stabilizing and destabilizing agents (ECCD on or inside the $q=1$ surface, respectively) were used to pace sawtooth oscillations, permitting precise control of their period. Locking of the sawtooth period to a pre-defined ECRH modulation period was also demonstrated. Sawtooth control has permitted nearly failsafe NTM prevention when combined with backup NTM stabilization by ECRH.
\end{abstract}

\section{Introduction}

The Tokamak à Configuration Variable (TCV) is a major European fusion facility fully embedded within an institution of higher learning. It is operated intensively by a crew whose scientific component is divided evenly between graduate students and senior staff and includes a large proportion of external collaborators from numerous institutions in Europe and beyond. The scientific program is deliberately endowed with a high degree of flexibility, allowing it to respond quickly to new challenges from theory and to novel experimental proposals, which are accommodated alongside the longer-term, goal-driven campaign plan.

\footnotetext{
${ }^{1}$ S.Alberti, H. Anand, M. de Baar, ${ }^{\mathrm{a}, \mathrm{b}}$, R. Behn, P. Blanchard, A. Bortolon ${ }^{\mathrm{c}}$, F.H. Braunmüller, S. Brunner, Y. Camenen $^{\text {d }}$, G.P. Canal, S. Coda, N. Cruz ${ }^{\mathrm{e}}$, L. Curchod, J. Decker ${ }^{\mathrm{f}}$, C.A. de Meijere, B.P. Duval, W.W. Eshetu, E. Fable $^{\mathrm{g}}$, A. Fasoli, L. Federspiel, F. Felici, I. Furno, S. Gnesin, T.P. Goodman, J.P. Graves, P. Hennequin ${ }^{\mathrm{h}}$, J.-Ph. Hogge, Z. Huang, B. Joye, J. Kamleitner, A. Karpushov, D. Kim, N. Kirneva ${ }^{i}$, A. Krämer-Flecken' ${ }^{j}$, B. Labit, M. Lauret $^{\mathrm{a}, \mathrm{b}}$, E. Lazzaro ${ }^{\mathrm{k}}$, H.B. Le, F. Li, J.B. Lister, X. Llobet, C. Marini, J. Marki, Y. Martin, S. Medvedev ${ }^{\mathrm{m}}$, J.-M. Moret, T. Morgan ${ }^{\mathrm{b}}$, S. Nowak ${ }^{\mathrm{k}}$, Y. Peysson ${ }^{\mathrm{f}}$, A. Pitzschke, A. Pochelon, L. Porte, H. Reimerdes, J.A. Romero $^{n}$, J.X. Rossel, O. Sauter, P. Savrukhini , A. Scaggion ${ }^{\circ}$, Ch. Schlatter, M.G. Sevillano ${ }^{p}$, M. Silva, J. Sinha, B. Tál ${ }^{\mathrm{q}}$, G. De Temmerman ${ }^{\mathrm{b}}$, D. Testa, G. Tonetti, M.Q. Tran, G. Vandersteen ${ }^{\mathrm{r}}$, G. Véres ${ }^{\mathrm{q}}$, L. Vermare ${ }^{\mathrm{h}}$, W.A.J. Vijvers, V. Vuille, D. Wágner, H. Weisen, G. Witvoet ${ }^{\mathrm{a}, \mathrm{b}}$, A. Zhuchkova, J. Zielinski ${ }^{\mathrm{b}}$.

${ }^{\mathrm{a}}$ TUe, The Netherlands; ${ }^{\mathrm{b}}$ DIFFER, The Netherlands; ${ }^{\circ} \mathrm{UC}$ Irvine, US; ${ }^{\mathrm{d}} \mathrm{CNRS} / \mathrm{AMU}$, France; ${ }^{\mathrm{e}}$ IST, Portugal; ${ }^{\mathrm{f}}$ CEA, France; ${ }_{\mathrm{g}}^{\mathrm{g}} \mathrm{IPP}$ Garching, Germany; ${ }^{\mathrm{h}} \mathrm{LPP}$, France; ${ }^{\mathrm{i}} \mathrm{RRC}$ Kurchatov, Russian Federation; ${ }^{\mathrm{j}}$ Forschungszentrum Jülich, Germany; ${ }^{\mathrm{k}} \mathrm{IFP}-\mathrm{CNR}$, Italy; ${ }^{\mathrm{m}}$ Keldysh Institute, Russian Federation; ${ }^{\mathrm{n}} \mathrm{CIEMAT}$, Spain; ${ }^{\circ}$ Consorzio RFX, Italy; ${ }^{\mathrm{p}} \mathrm{EHU}$, Spain; ${ }^{\mathrm{C}} \mathrm{IPNP}$, Hungary; ${ }^{\mathrm{r}} \mathrm{VU}$ Brussels, Belgium.
} 
TCV [1] is a conventional aspect ratio tokamak (major and minor radii 0.88 and $0.25 \mathrm{~m}$, respectively) with a vacuum toroidal magnetic field of $1.5 \mathrm{~T}$ and an air-core transformer able to drive a plasma current up to $1 \mathrm{MA}$. A highly elongated vacuum vessel accommodates plasmas of elongation up to 2.8, and a set of 16 independently powered shaping and positioning coils - supplemented by two fast internal coils for vertical stabilization - are at the root of a unique shaping capability that encompasses negative triangularity [2] $(-0.7<\delta<1)$, second-order X-points (as in the "snowflake" divertor configuration [3]), highly up-down asymmetric plasmas [4], and - potentially - doublets [5]. Auxiliary heating is provided by Electron Cyclotron Resonance Heating (ECRH), supplied by nine 0.5-MW gyrotrons (six at the $2^{\text {nd }}(\mathrm{X} 2)$ and three at the $3^{\text {rd }}(\mathrm{X} 3)$ harmonic, respectively 82.7 and $\left.118.0 \mathrm{GHz}\right)$ to seven launchers with independent real-time steering capabilities. Fully non-inductive operation with Electron Cyclotron Current Drive (ECCD) is routinely performed up to a current of $210 \mathrm{kA}$ [6], and a wide range of regimes is accessible, including internal transport barriers with reverse magnetic shear [7] and quiescent ELM-free H-modes [8], spanning a ratio of electron to ion temperature from 1 to 20 . The dominant electron heating is an effective simulator of aspects of ITER [9] alpha-particle heating, while the overall flexibility encourages investigations of alternative paths to commercial fusion. Both paths will be broadened further by planned upgrades, including additional ECRH sources and neutral beam injectors for direct ion heating.

This paper reports on the results of the 2011 campaign, which was followed by an extensive shutdown for the first ten months of 2012. Strong emphasis was placed on the development and demonstration of real-time control techniques, employing a new digital control system [10] and affecting virtually every scientific theme. The remainder of the paper is organized indeed by theme, with $\mathrm{H}$-modes and ELMs discussed in section 2, the snowflake divertor in section 3, confinement and transport studies in section 4, ECRH physics in section 5, discharge optimization in section 6, and MHD instabilities in section 7. Concluding remarks are offered in section 8 .

\section{H-mode and ELMs}

In H-mode, the reference operating scenario for ITER, Edge Localized Modes (ELMs) constitute a major concern as they may subject the plasma facing vessel components to bursts of excessive power, potentially leading to a rapid deterioration of the wall integrity and to reactor shutdown [11]. Accordingly, the fusion community is engaged in a vigorous search for methods of ELM control, with the aim of regulating the energy release per ELM [12]. On TCV the potential of edge-deposited ECRH power to affect the properties of ELMs was recently investigated, with encouraging results. These experiments targeted specifically the more virulent type-I ELMs, which were produced in a single-null diverted plasma, heated by $1 \mathrm{MW}$ core X3 ECRH. The high absorption efficiency of the X2 waves was then exploited to deposit power locally in the edge region (Fig. 1). An important finding is that the frequency of type-I ELMs increases and their relative energy loss decreases as power is deposited closer to the plasma boundary, which runs contrary to the intrinsic power scaling since the effective absorbed power simultaneously decreases [13]. This not only suggests a possible mitigation technique, it also puts into question the current understanding of ELM dynamics, as it has generally been accepted that the power scaling is independent of heating location.

Successful ELM pacing has been demonstrated by following individual ELM detection with an ECRH power drop before turning the power back up, which eventually triggers the next ELM. The resulting ELM frequency still satisfies its known scaling with average power, so that it can be decreased by prolonging the pre-programmed cut duration, and vice versa. Crucially, however, stimulating the occurrence of individual ELMs significantly reduces the 


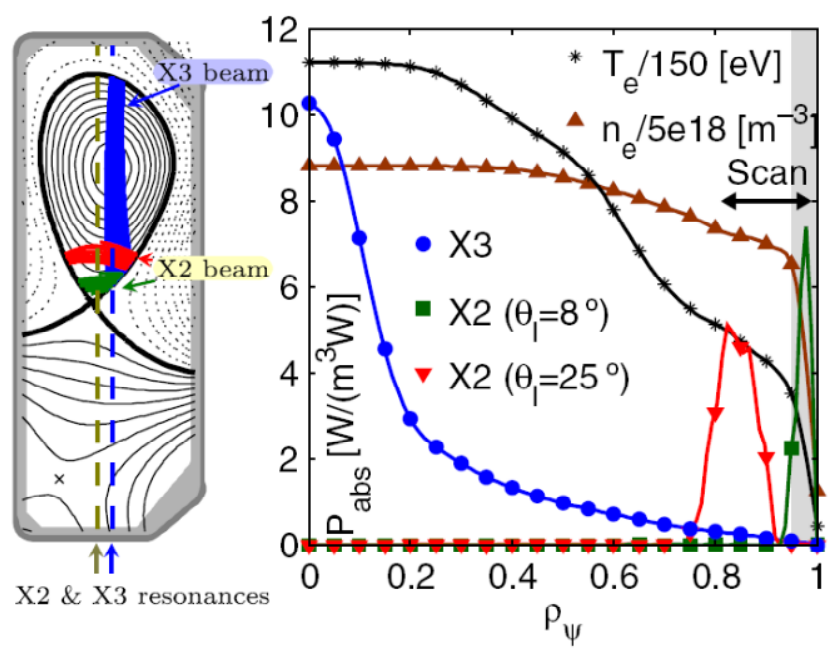

FIG. 1. (Left) Flux-surface contours and ray trajectories for $X 3$ and $X 2$ (in two possible configurations); (Right) X3 and X2 power deposition profiles (calculated by ray tracing), electron density and temperature profiles from Thomson scattering. (Reproduced from [13].) dispersion of the period (standard deviation as low as $3 \%$ ) compared with continuous heating; furthermore, the dispersion decreases with increasing ELM frequency (hence with X2 power) [14] (Fig. 2). Additionally, a desired ELM frequency can be precisely dictated through real-time adjustments of the duration of the low-power phase, again with comparably low dispersion [15]. The dispersion does not increase even when the duration of the power cut is varied - along a pre-programmed sequence - after each ELM (Fig. 3), with the ELM period remaining faithful to its dependence on the average power [14]. There is therefore no apparent memory in the system, i.e., each ELM is independent of the previous one. A simple power integrator model, positing a pedestal energy threshold for ELM triggering, can reproduce the salient features of the results; in particular, the dispersion in the period can be explained by an uncertainty in the energy threshold, which naturally impacts the trigger time less if the instantaneous power is higher [14].

The unique capability of TCV to sustain negative-triangularity plasmas was recently extended for the first time to H-mode. Teardrop-shaped, single-null diverted configurations can be heated efficiently and steadily with both $\mathrm{X} 2$ and $\mathrm{X} 3 \mathrm{ECRH}$, in a

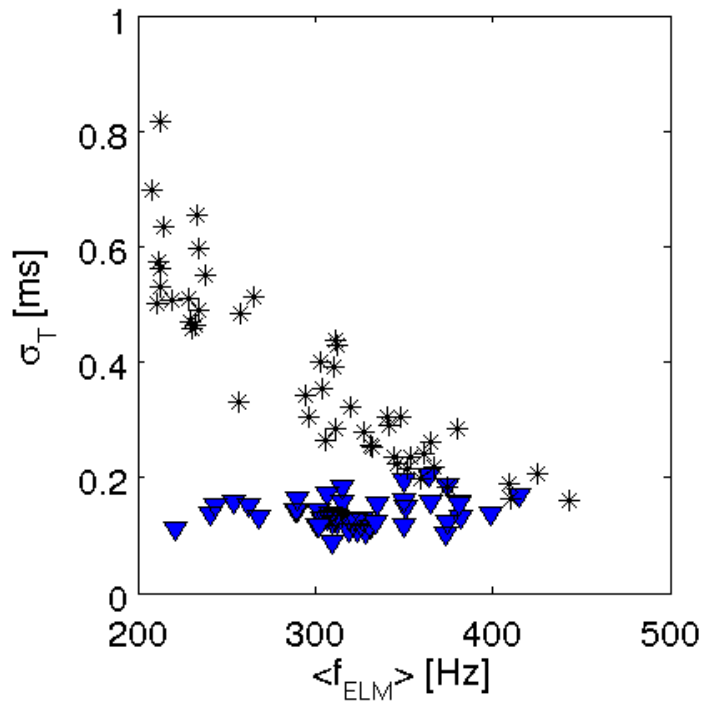

FIG. 2. Standard deviation of ELM period vs average ELM frequency, for stationary heating (stars) and real-time control (triangles).

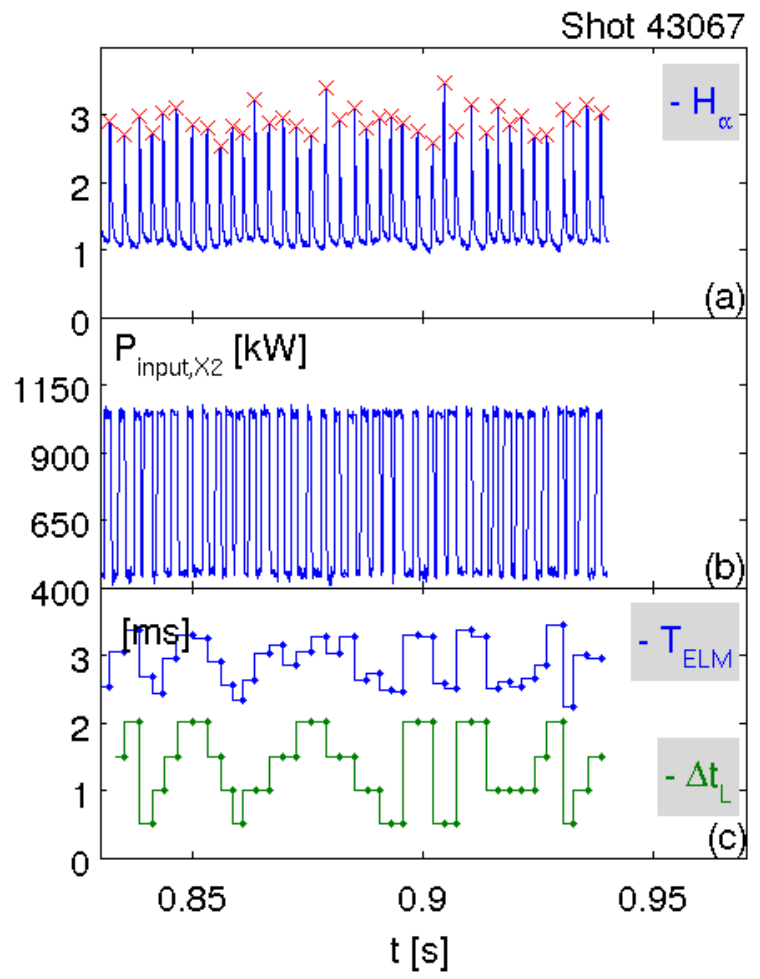

FIG. 3. (a) ELMs as seen on $H_{\alpha}$ signal; (b) X2 power trace; (c) measured ELM period $\left(T_{E L M}\right)$ and power cut duration $\left(\Delta t_{L}\right)$ determined by $a$ pre-programmed ELM-to-ELM random sequence. 
type-I ELMing H-mode, throughout a scan of the triangularity from +0.2 to -0.2 (Fig. $4 \mathrm{a}$ ). A striking increase in ELM frequency, accompanied by a corresponding reduction in energy release per ELM, is observed in going to negative triangularity (Fig. 4b) [16]. This is a form of ELM mitigation in an alternative configuration not available to ITER, although it is accompanied by a worsening of confinement - in marked contrast with the L-mode regime, where confinement is better for $\delta<0$ than for $\delta>0$ [2].

Fundamental studies of the power threshold for the L- to H-mode transition in ITERcompatible plasma geometries have progressed in parallel, focusing in particular on its dependences on the ion species and on the length of the divertor leg, open questions that must be addressed in order to formulate more confident predictions for ITER. Ohmic H-modes are achievable in TCV with hydrogen, deuterium, and helium plasmas when the ion $\nabla \mathrm{B}$ drift is directed towards the X-point; however, while the power threshold is similar for $\mathrm{H}$ and $\mathrm{He}$, it is found to be lower for D. In similar conditions, but with the addition of ECRH power, the vertical distance of the X-point from the vessel floor - and thus the length of the divertor leg was varied systematically in D plasmas, evincing a monotonic increase of the threshold power with the distance of the X-point to the strike point [17]. These experiments also confirmed the non-monotonic dependence of the threshold on line-averaged density, displaying a minimum at $\sim 3.6 \times 10^{19} \mathrm{~m}^{-3}$.

\section{Snowflake divertor studies}

The snowflake (SF) divertor, a configuration predicated on an X-point with vanishing poloidal field gradients [3], is now well recognized as a viable operating configuration thanks to its successful demonstration on TCV [18-19]. Its ability to reduce the energy and particle flux to an appropriately designed divertor, due to the doubling of the number of divertor legs and to the increased flux expansion, is well documented $[18,20]$.

As the SF proper occupies a vanishingly small parameter region, the only experimentally practicable configurations are the so-called SF+ and SF-, possessing two neighboring $\mathrm{X}$ points. The distance between the X-points normalized to the plasma minor radius, $\sigma$, provides a convenient parametrization of the continuum between the SF and a conventional singlenull (SN) diverted topology. A systematic scan of $\sigma$ was recently carried out to document the transition between these regimes and the activation of the two additional strike points in both $\mathrm{L}-$ and $\mathrm{H}$-mode. In $\mathrm{H}-$ mode, the frequency of type-I ELMs is found to increase with $\sigma$, with a less-thanproportional decrease in fractional energy release, resulting in a favorable
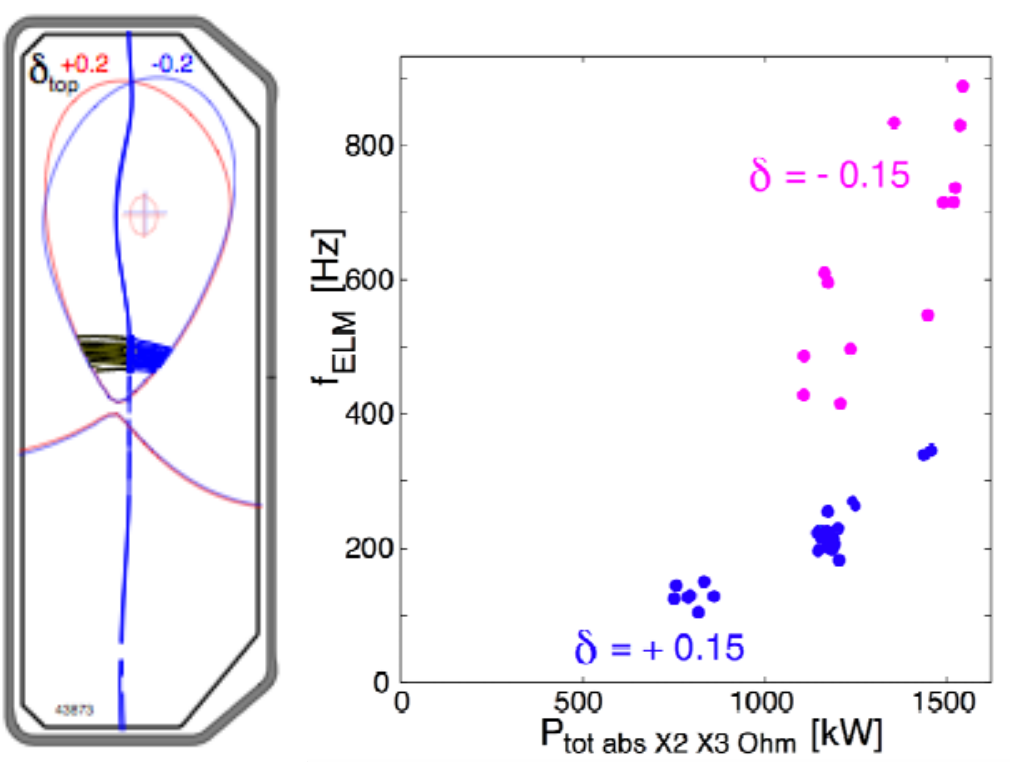

FIG. 4. (Left) Flux-surface contours with ray trajectories for edgedeposited X2 for positive and negative triangularity teardropshaped plasmas; (Right) Type-I ELM frequency vs total input power for positive and negative triangularity. (Reproduced from [16].) 
scaling of average heat flux for the SF configuration [19,21]. The lowest $\sigma$ values in this scan, however, were accompanied by a transition to type-III ELMs, with higher frequency and degraded confinement.

The particle flux to the walls is monitored with Langmuir probe arrays on the floor and on the central column, covering the two secondary strike points as well as one of the two primary ones. The probes are operated at a constant negative voltage $-100 \mathrm{~V}$, to measure the ion saturation current. The analysis, based on coherent averaging over ELM cycles, is complicated by shifts in the strike point positions, as well as by a 10\% drop in $\sigma$ at each ELM crash. However, it is clear that the secondary strike points begin to be activated for $\sigma \leq 1.2$ (Fig. 5), and that for $\sigma=0.4$ the flux to them is of order $30 \%$ of the flux to the primary strike points [21,22]. The delay between the ELM crash and the particle flux peak is consistent with parallel propagation at the ion sound speed. It should be noted, by contrast, that in L-mode the secondary strike point activation is only observed for $\sigma<0.6$.

Edge radiation is measured by a tomographic array of 7 pinhole cameras with 20 AXUV detectors each, sensitive to photons in the $1 \mathrm{eV}-10 \mathrm{keV}$ range but with spectral gaps precluding a true bolometric measurement. The activation of the lower strike point at the ELM crash is clearly visible in the coherently averaged tomographic reconstruction for the lowest value of $\sigma$ (Fig. 6). The overall radiated fraction in the AXUV spectral range is $70 \%$
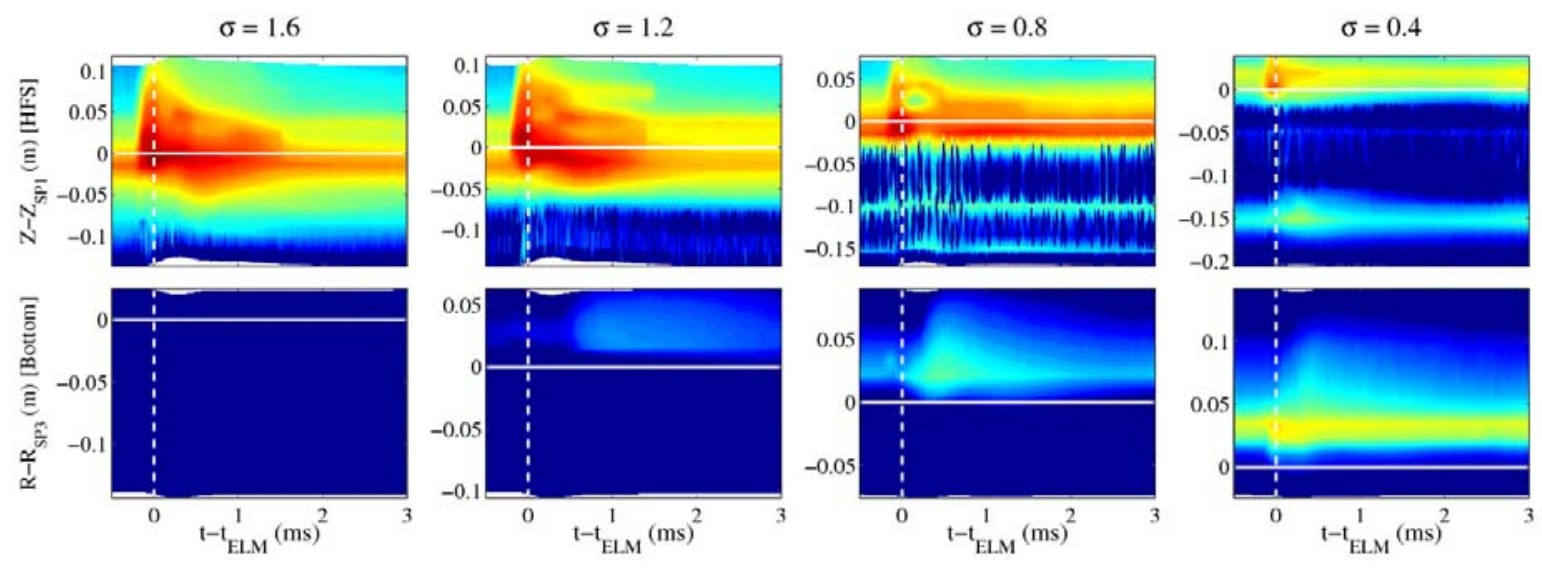

FIG. 5. Contour plots in space and time, coherently averaged over the ELM cycle ( $t_{E L M}$ denoting the ELM crash), of Langmuir probe ion saturation current, for a primary strike point on the inside wall (top) and a secondary snowflake strike point on the floor (bottom).

higher at $\sigma=0.4$ than at $\sigma=1.2$, an observation whose interpretation is complicated, however, by the different ELM types in the two cases [21]. The wall response is monitored by two fast framing $(>10 \mathrm{kHz})$ infrared cameras observing the floor and the inside wall. The activation of the lower strike points at the ELM crash is apparent in these measurements for $\sigma<0.6$, with heat loads peaking at $10 \mathrm{MW} / \mathrm{m}^{2}$ [22]. The heat flux to the primary strike point on the inside wall, as reconstructed from these measurements, drops by a factor 3.5 as $\sigma$ decreases from 2.4 to 0.4 [21]. While the heat flux profile is asymmetric around the primary strike point, as is generally observed in SN plasmas, it is symmetric around the lower strike point. This is qualitatively consistent with a recent model predicting that flux to this location for $\sigma \neq 0$ is governed by plasma convection driven by the toroidal curvature for $\beta_{\mathrm{p}}>1$ [23]. The lower $\sigma$ required for secondary strike point activation in L-mode is also consistent with this model, as $\beta_{\mathrm{p}}$ is lower in that regime. 


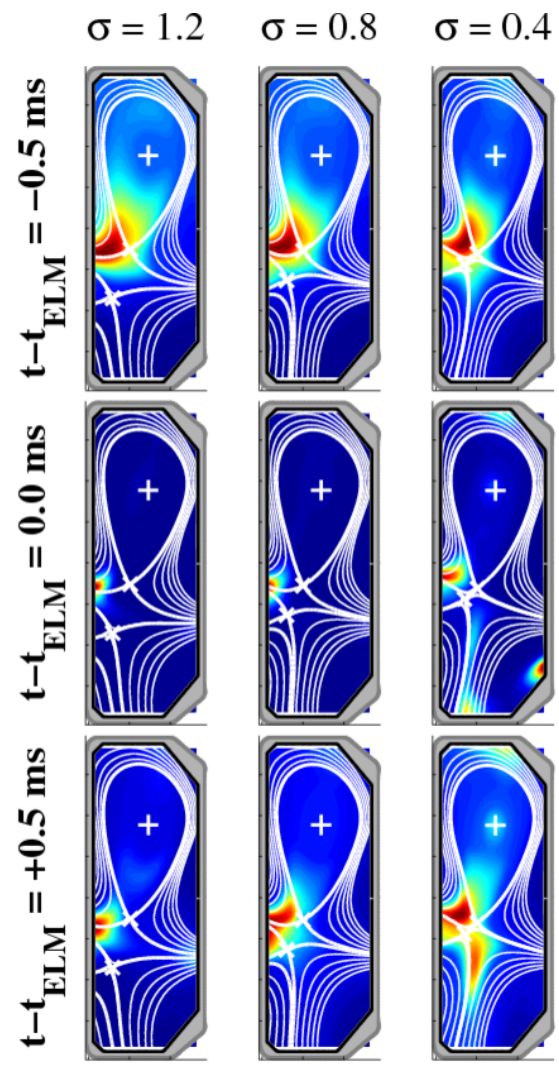

FIG. 6.

Tomographically reconstructed radiation as detected by AXUV array, coherently averaged over ELM cycle.

Langmuir probes on the low-field-side wall near the plasma midplane were employed to investigate intermittency in transport in Ohmic L-mode plasmas. Conditionally sampled ion saturation currents exhibit large spikes, indicating the presence of density blobs propagating towards the wall. These blobs are markedly less frequent in the SF than in the SN case, suggesting either faster parallel convection or blob suppression by the higher SF magnetic shear [24].

\section{Confinement and transport studies}

The versatility of the TCV ECRH system has prompted an exploration of the long-standing question of the origin of L-mode confinement degradation with auxiliary power, and more specifically of its possible dependence on the power deposition profile. If a significant dependence existed, it could be expected that an auxiliary power deposition profile closely replicating the Ohmic heating profile would restore the Ohmic level of confinement. Centrally peaked X2 ECRH was employed for this study. A scan of the ECRH deposition width was performed, from a minor radius HWHM of 0.1 to the Ohmic-like value 0.4 , yielding in each case a power scaling of the energy confinement time $\tau_{\mathrm{E}} \propto \mathrm{P}^{-0.73}$, consistent with ITER L-mode scaling [25, 26]. This result holds for different values of plasma current and also for negative triangularity, which is characterized by better overall confinement. The power deposition profile width can thus be ruled out as the cause of confinement degradation.

When the ECRH deposition is peaked off-axis, by contrast, the power degradation worsens $\left(\tau_{\mathrm{E}} \propto \mathrm{P}^{-0.9}\right)$. The crucial factor appears to be the fraction of power deposited outside the $q=1$ surface, as confirmed by an experiment using a single ECRH power source with the launcher swept from far outside the $q=1$ surface to the magnetic axis. Figure 7 shows that confinement improves sharply as the $q=1$ surface is crossed.

The strong observed dependence of confinement on triangularity and collisionality in TCV [2] has motivated inquiries into the behavior of the plasma turbulence that is thought to underlie anomalous transport. Preliminary investigations have been conducted using a 2channel correlation ECE diagnostic, measuring electron temperature fluctuations. An increase in collisionality by a factor 2.4 is seen to result in a decrease of the radial correlation length by a factor 2.7. At equal collisionality, a dramatic collapse in fluctuation amplitude is observed in going from positive to negative triangularity $(\delta= \pm 0.4)$ [28]. These measurements will be extended and completed after an imminent upgrade of the diagnostic and will be complemented by data from a new tangential phase contrast imaging diagnostic.

Explorations of momentum transport have been ongoing on TCV for several years. Spontaneous rotation is observed to occur even in the absence of any significant direct external momentum input [29]. Its properties are studied with a charge exchange recombination spectroscopy (CXRS) diagnostic paired with a low-power neutral beam and tuned to the $C^{6+}$ impurity line. Progressive hardware additions have resulted in a complete set 
of measurements of both toroidal and poloidal rotation speed. The latter, however, while crucial for a correct determination of the radial electric field, is generally too small to be measured accurately. An alternative, indirect method has recently been developed, based on the fundamental property of incompressible flows that the inboard to outboard variation of the toroidal rotation speed is proportional to the poloidal rotation speed [30]. Because this proportionality factor is approximately equal to four times the safety factor $q$, and $4 q » 1$, this procedure effectively amplifies the rotation, provided the toroidally viewing chords have a complete inboard to outboard coverage, as is the case in TCV. The technique has been applied to TCV L-mode plasmas in the banana-plateau collisionality regime, and carbon poloidal flow speeds of order $0.5-2.5 \mathrm{~km} / \mathrm{s}$ in the electron diamagnetic drift direction have been inferred, in fair agreement with neoclassical predictions [30].

\section{ECRH physics}

The ITER design includes a substantial ECRH power capability for the main purpose of profile control and instability suppression, rather than for bulk heating or current drive. The power invested for control purposes is generally not efficiently confined and thus impacts the reactor power balance unfavorably. Maximizing the absorption efficiency is therefore paramount, and plasma wave physics translates this requirement to that of optimal polarization. An attendant benefit of enhanced absorption is a minimization of lost radiation, which can potentially damage vacuum components such as windows. Real-time polarization control is thus planned for ITER, and fast polarizers are currently being tested on TCV for this purpose. A crucial element in this development is the establishment of a robust ECRH absorption measurement method. As modulation techniques have been shown to be unreliable in the presence of sawtooth oscillations, an alternative approach was investigated on TCV based on the detection of escaped radiation by microwave detectors [31]. This serves also as a fundamental validation of ray-tracing codes that are routinely used to plan ECRH experiments.

In these tests, a calibrated Schottky detector was coupled through a fast universal polarizer to a receiver antenna that is an exact replica of an X2 launcher, allowing the polarization and propagation direction of the measured radiation to be selected at will. A single X2 source was employed in pulsed mode, again with selectable orientation and polarization, and the geometry was optimized for detection of the waves after two passes through the plasma and one reflection from the inside wall. In general, the launched beam contains a combination of $\mathrm{X}$ - and O-mode, each of which generates $\mathrm{X}$ - and O-mode components upon reflection, resulting in four detectable beam components. This complex scenario was studied with launcher and receiver angle scans as well as polarization scans. Figure 8 shows an example of 
a launcher angle scan for O-mode transmission, with a fit to the measurements based on the calculated properties of the beam, and only the absolute amplitude and peak angle left as fit parameters.

The excellent agreement at once provides confidence in the calculations and confirms the applicability of the technique [31].

A concrete application planned for TCV is a fundamental study of quasilinear effects of ECRH on the electron velocity distribution function. Two competing effects are expected to dominate: increased plasma transparency from distribution flattening, and increased opacity from an enhanced suprathermal population. These effects can best be evidenced experimentally in conditions of medium optical thickness $(\sim 0.6)$, and quasilinear FokkerPlanck calculations can accordingly be verified quantitatively by the lost radiation method [31].

Fokker-Planck simulations were also employed in a detailed study of the conditions for synergy between $2^{\text {nd }}$ and $3^{\text {rd }}$ harmonic heating at the same frequency in TCV, shedding new light on the complex velocity-space dynamics involved. An attempt to measure the effect experimentally was, however, unsuccessful as it proved impossible to disentangle from other, unrelated effects [32].

ECRH and ECCD physics in TCV is intimately tied to the dynamics of suprathermal electrons. A novel tomographic hard X-ray (HXR) spectrometer is being developed for TCV with the aim of performing the first $2 \mathrm{D}$ analysis of suprathermal bremsstrahlung emission in a noncircular plasma. The first of four cameras is already in operation and has allowed us to verify for the first time the predicted HXR toroidal asymmetry in the presence of ECCD and to characterize the suprathermal electron response to sawtooth oscillations. Advanced pulse detection algorithms have been expressly developed for postprocessing data from this diagnostic in high-flux conditions close to pulse pile-up [33].

\section{Tokamak discharge optimization}

This campaign has witnessed the elaboration of an entire new profile control methodology relying on real-time modeling to supplement diagnostic information. This is proposed as a

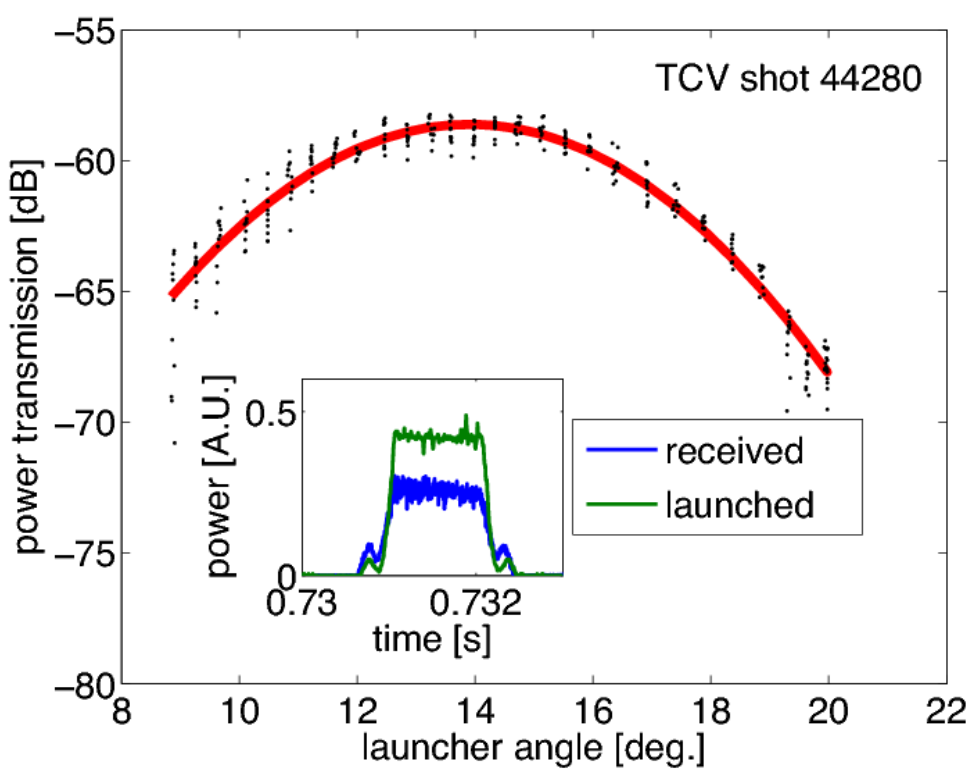

FIG. 8. Lost radiation power vs ECRH launcher angle for short repetitive pulses in O-mode (black) and fitted curve (red). The pulse shape detail is shown in the inset. new paradigm for the control of present and especially future devices, utilizing prior physics knowledge to provide quantities not readily available to measurement. The integration of simulations with measurements is performed seamlessly by adopting a state observer approach, which also facilitates the incorporation of further variables as they become available. The concrete incarnation of this methodology, the RApid Plasma Transport simulatOR (RAPTOR) [34], implements a current transport model to reconstruct the current density profile, supplying 
quantities such as the bootstrap current fraction, the safety factor and magnetic shear profiles, and the loop voltage profile. The code has been benchmarked on TCV discharges and directly employed for initial joint control of the internal inductance (simulated) and central electron temperature (measured), as shown in Fig. 9. The code is programmed in SIMULINK, which is then converted to $\mathrm{C}$ code and compiled on the nodes of the TCV digital control system [10]. In this implementation, the current diffusion PDE is solved in steps of $1 \mathrm{~ms}$, i.e., approximately 100 times faster than the characteristic current diffusion time; as this time will be considerably longer in most future fusion devices, this constitutes a robust proof of principle.

We have also turned our attention to the Ohmic transformer, a powerful actuator for current profile control, which can lessen the auxiliary power requirements in a reactor. In the spirit of improving tokamak economics, a general nonlinear optimization technique for the discharge evolution was developed, based on satisfying known constraints and on minimizing a practical cost function [36]. The calculations are based on a predictive version of RAPTOR, which incorporates an energy transport simulator. The first proposed application tackles the ramp-up problem, aiming at reaching a stationary state rapidly with minimal flux consumption [35].

A parallel development focused specifically on manipulating the current profile during the ramp-up using the Ohmic transformer. Using this approach, internal inductance control was achieved in TCV, from $30 \mathrm{~ms}$ after breakdown to the end of the discharge, by deploying a sliding-mode control algorithm [37], based on a lumped-parameter model of the transformer, readily adaptable to other tokamaks [38]. Extensions to multi-parameter control, adding a recently tested $\beta$ observer [39], are planned for the future.

In the absence of active cryopumping, effective density control has often been difficult with the original analogue PID plasma control system. A new digital algorithm has therefore now

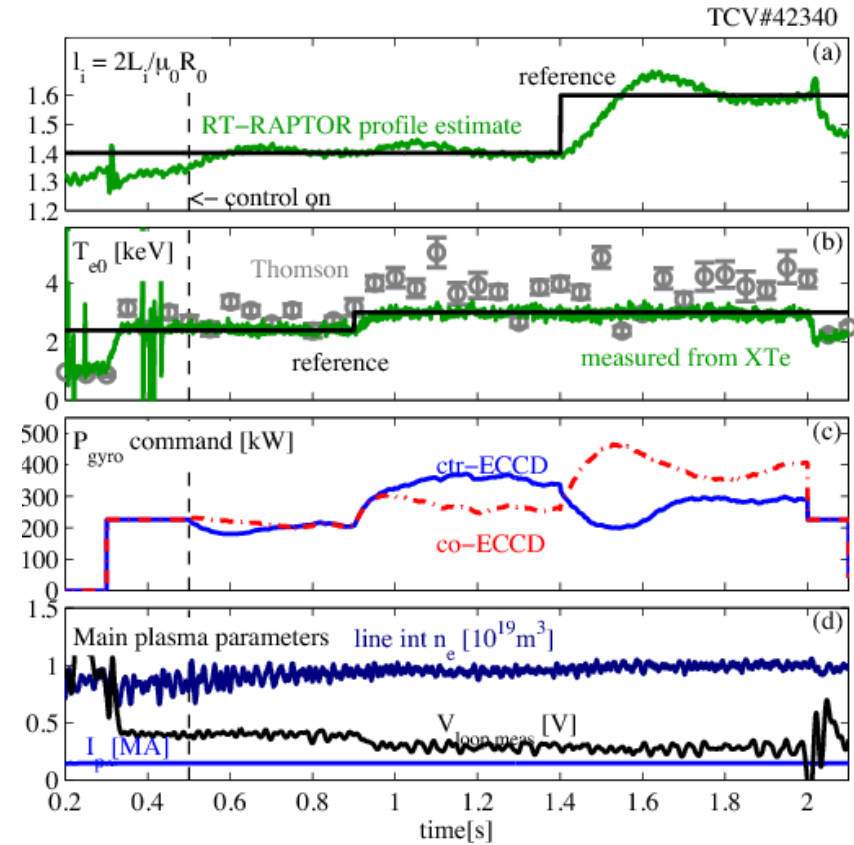

FIG. 9. Real-time control of (a) internal inductance and (b) central electron temperature. Two ECRH sources were used as actuators, applying co- and counter-ECCD, respectively, near the magnetic axis (c). Additional plasma parameters are shown in (d). been implemented, based on a PI controller but incorporating an antiwindup mechanism, asymmetric gain to address the lack of active pumping, and low-pass filtering for noise reduction. The algorithm has operated successfully on a number of TCV discharges [40].

\section{MHD instabilities}

Experiments are ongoing to explore the density disruption limit in TCV with and without ECRH, as well as confinement in the proximity of the limit. In density ramps in Ohmic plasmas, the limit is reached close to the Greenwald value $\mathrm{n}_{\mathrm{GW}}$ at low current $(110 \mathrm{kA})$, whereas disruptions occur at $0.63 \times \mathrm{n}_{\mathrm{GW}}$ at $200 \mathrm{kA}$. These discharges systematically exhibit a disappearance of sawtooth oscillations shortly before the disruption, concomitantly with density peaking and followed by the destabilization of $m=1,2, n=1$ modes 
[26]. Although application of ECRH to prevent sawtooth stabilization is effective in raising the limit density, the complex chain of events is not fully understood and the generality of the observations cannot be confirmed without further experiments.

Neoclassical tearing modes (NTMs) are well known to limit performance or lead to disruptions and are accordingly actively investigated in view of ITER operation. An experiment was conducted on TCV to address the unresolved question of whether NTMs can be triggered in the absence of a seed island. In discharges without sawteeth or ELMs, $m=2$, $n=1$ NTMs were found to be destabilized after the application of central ECRH and ECCD and after the appearance of a conventional $m=3, n=2$ tearing mode. The toroidal rotation speed is reduced first by the application of auxiliary power, then by the $(3,2)$ mode, and it is speculated that this is related to the eventual destabilization of the NTM, mediated by the ion polarization current [41].

The best-known trigger mechanism for NTMs is provided by the crash of a long-period sawtooth. This, too, has been investigated on TCV by analysis of integrated magnetic data. For both $(3,2)$ and $(2,1)$ mode excitation, it is found that a seed island forms within tens of $\mu \mathrm{s}$ of the sawtooth crash. The subsequent island development is consistent with the modified Rutherford equation. The magnetic perturbation induced by the sawtooth crash decreases with increasing edge safety factor, and the threshold in sawtooth period for NTM destabilization correspondingly increases [42].

Recent literature is rich with experimentation in the control of sawtooth oscillations [43], motivated primarily by the desire to prevent NTM triggering. A similar technique to that described in section 2 for ELM control was in fact used originally with complete success to control the sawtooth period on TCV. A stabilizing, rather than destabilizing, agent is used in this case. Here, ECCD is applied near the $q=1$ surface for a set duration after the detection of a sawtooth crash; once the power is turned off, the next sawtooth is triggered after a short and remarkably reproducible interval. As a result, the period of individual sawteeth can be prescribed and varied at will with nearly complete precision, as illustrated by Fig. $10[15,44]$. Pacing by destabilization, similar to the ELM control scenario, has also been achieved, by turning on ECRH inside the $q=1$ surface a prescribed time after each sawtooth crash. In the presence of a concomitant stabilizing actuator - ECCD on the $q=1$ surface - this scheme emulates the control scenario required in ITER, where stabilization is expected from energetic $\alpha$ particles. Finally, locking of the sawtooth period to a pre-defined ECRH modulation period has also been demonstrated within a slightly narrower period range [45], consistent with control-theory predictions [46].

NTM stabilization by ECCD has been shown on TCV, as elsewhere, to require the power deposition to be within one-half beam width of the island location. However, the simple device of sweeping the deposition radius across the region of interest by launcher aiming, after a mode is detected, has proven effective on TCV [47]. The sweep is halted once the mode disappears. ECCD power applied to the relevant rational surface in the absence of a mode has also been found to be effective in reducing the probability of NTM triggering by sawtooth crashes. Magnetic measurements have revealed that this scheme works by reducing the seed island size [42]. A multi-launcher integrated scheme for $m=3, n=2 \mathrm{NTM}$ prevention has been tested successfully whereby (a) the sawtooth period is kept below a set value; (b) moderate ECCD power is applied to the $q=3 / 2$ surface just ahead of the (precisely known) sawtooth crash time to prevent NTM triggering; (c) if an NTM is nonetheless detected, the $q=3 / 2$ power is increased enough to stabilize the mode. This double-backup tool set has proven robust and effectively failsafe $[47,48]$. 
TCV\#42297

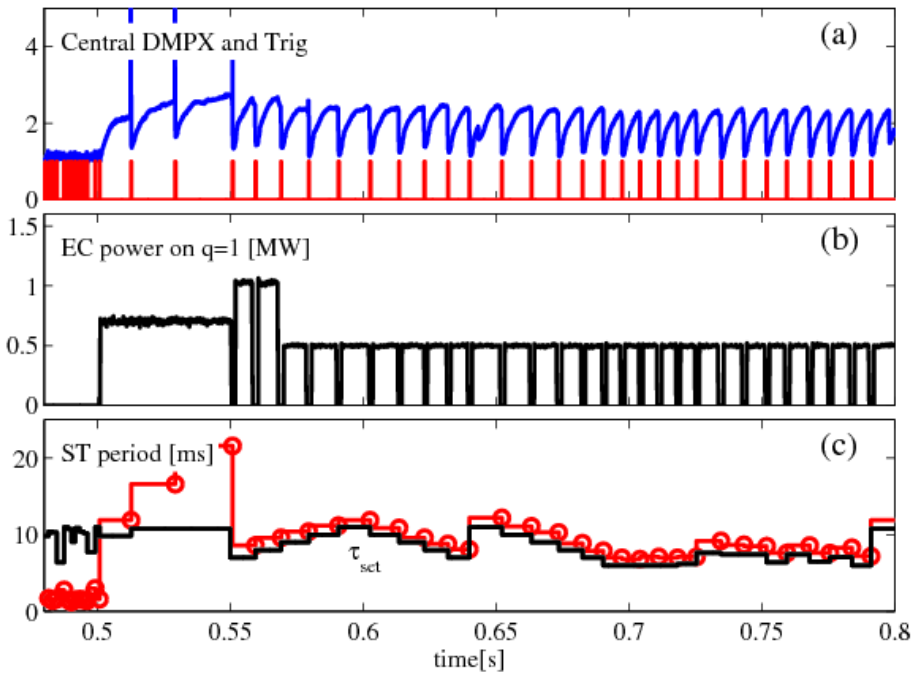

FIG. 10. Real-time control of sawtooth period from time 0.55 s: (a) soft $X$-ray signal and detected sawtooth crash times; (b) ECRH power; (c) requested (black) and achieved (red) sawtooth period. (Reproduced from [42].)

\section{Conclusions and outlook}

A diverse and demanding research program has driven an intensive operation campaign on TCV during the past two years, to address physics issues and develop tools for ITER and for the longer-term goals of nuclear fusion. Highlights include the elaboration and demonstration of robust methods of integrated MHD instability control (ELMs, sawteeth, NTMs) and discharge evolution control, progress in the understanding of the advanced snowflake divertor configuration pioneered by TCV, parametric explorations of $\mathrm{H}$-mode behavior, and fundamental studies of transport, MHD, and ECRH waveparticle interaction physics.

The existing auxiliary electron heating power is due to increase further and to be supplemented by MW-level neutral beam heating in the near future, with a view to expanding the accessible parameter space and its overlap with the reactor realm.

New diagnostics are continuously being developed, the latest additions to the roster being a spectroscopic hard X-ray (HXR) multi-chord camera [33], a vertical ECE system [49], and a laser phase-contrast apparatus for fluctuation imaging. The development that has had arguably the greatest impact is a new digital real-time control system, based on a readily scalable cluster of Linux PC nodes linked by a reflective-memory network [10]. This has affected virtually every area of research, and its flexibility is likely to encourage all newly acquired physical understanding to be translated into new control tools.

\section{Acknowledgment}

This work was supported in part by the Swiss National Science Foundation.

\section{References}

[1] F. Hofmann et al, Plasma Phys. Control. Fusion 36 (1994) B277.

[2] Y. Camenen et al, Nucl. Fusion 47 (2007) 510.

[3] D.D. Ryutov, Phys. Plasmas 14 (2007) 064502.

[4] Y. Camenen et al, Phys. Rev. Lett. 105 (2010) 135003.

[5] F. Hofmann et al, Proc. $23^{\text {rd }}$ EPS Conf. on Control. Fusion and Plasma Phys., Kiev, 1996, vol. I, p. a036.

[6] S. Coda et al, Plasma Phys. Control. Fusion 42 (2000) B311.

[7] S. Coda et al, Phys. Plasmas 12 (2005) 056124.

[8] L. Porte et al, Nucl. Fusion 47 (2007) 952.

[9] ITER Technical Basis, ITER EDA Documentation Series No 24, IAEA, Vienna (2002).

[10] J.I. Paley et al, Proc. 17th IEEE-NPSS Real Time Conference, RT10, Lisboa, Portugal (2010).

[11] A. Loarte et al., J. Nucl. Mater., 313-316 (2003) 962.

[12] Y. Liang, Fusion Sci. Technol. 59 (2011) 586. 
[13] J.X. Rossel et al, Nucl. Fusion 52 (2012) 032004.

[14] J.X. Rossel, Ph.D. thesis 5311, EPFL, Lausanne (2012).

[15] B.P. Duval et al, this conference, EX/1-2.

[16] A. Pochelon et al, Plasma and Fusion Research, to be published (2012).

[17] A. Scaggion et al, Proc. $39^{\text {th }}$ EPS Conf. on Control. Fusion and Plasma Phys., Stockolm, 2012, Europhys. Conf. Abstr. 36F (2012) (P-2.093).

[18] F. Piras et al, Plasma Phys. Control. Fusion 51 (2009) 055009.

[19] F. Piras et al, Phys. Rev. Lett. 105 (2010) 155003.

[20] V.A. Soukhanovskii et al, Phys. Plasmas 19 (2012) 082504.

[21] W.A.J. Vijvers et al, this conference, EX/P5-22.

[22] B. Labit et al, Proc. $39^{\text {th }}$ EPS Conf. on Control. Fusion and Plasma Phys., Stockolm, 2012, Europhys. Conf. Abstr. 36F (2012) (P-5.091).

[23] D.D. Ryutov, Contrib. Plasma Phys. 52 (2012) 539.

[24] B. Labit et al, Proc. $38^{\text {th }}$ EPS Conf. on Control. Fusion and Plasma Phys., Strasbourg, 2011, Europhys. Conf. Abstr. 35G (2011) (P-2.076).

[25] N. Kirneva et al, Plasma Phys. Control. Fusion 54 (2012) 015011.

[26] N. Kirneva et al, this conference, EX/P3-05.

[27] P.H. Rebut et al, Proc. $12^{\text {th }}$ Int. Conf. on Plasma Physics and Controlled Nuclear Fusion Research, Nice, 1988, vol. 2, p. 191, IAEA, Vienna (1989).

[28] L. Porte et al, Proc. $17^{\text {th }}$ Joint Workshop on ECE and ECRH (EC-17), Deurne, 2012, EPJ Web of Conferences 32 (2012) 03007.

[29] B.P. Duval et al, Phys. Plasmas 15 (2008) 056113.

[30] A. Bortolon et al, submitted to Nucl. Fusion (2012).

[31] T.P. Goodman et al, this conference, EX/P6-08.

[32] S. Gnesin et al, Plasma Phys. Control. Fusion 54 (2012) 035002.

[33] J. Kamleitner et al, Proc. $39^{\text {th }}$ EPS Conf. on Control. Fusion and Plasma Phys., Stockolm, 2012, Europhys. Conf. Abstr. 36F (2012) (P-1.010).

[34] F. Felici et al, Nucl. Fusion 51 (2011) 083052.

[35] F. Felici et al, this conference, EX/P3-12.

[36] F. Felici and O. Sauter, Plasma Phys. Control. Fusion 54 (2012) 025002.

[37] J.A. Romero et al, this conference, EX/P4-35.

[38] J.A. Romero et al, Nucl. Fusion 52 (2012) 023019.

[39] M.G. Sevillano et al, Proc. 50 ${ }^{\text {th }}$ IEEE CDC-ECC, Orlando, 2011, p. 7536.

[40] W.A.J. Vijvers et al, Proc. $39^{\text {th }}$ EPS Conf. on Control. Fusion and Plasma Phys., Stockolm, 2012, Europhys. Conf. Abstr. 36F (2012) (P-1.066).

[41] E. Lazzaro et al, this conference, EX/P4-32.

[42] G.P. Canal et al, Proc. $39^{\text {th }}$ EPS Conf. on Control. Fusion and Plasma Phys., Stockolm, 2012, Europhys. Conf. Abstr. 36F (2012) (P-5.076).

[43] I.T. Chapman, Plasma Phys. Control. Fusion 53 (2011) 013001.

[44] T.P. Goodman et al, Phys. Rev. Lett. 106 (2011) 245002.

[45] M. Lauret et al, Nucl. Fusion 52 (2012) 062002.

[46] G. Witvoet et al, Nucl. Fusion 52 (2012) 074005.

[47] F. Felici et al, Nucl. Fusion 52 (2012) 074001.

[48] F. Felici et al, Proc. $17^{\text {th }}$ Joint Workshop on ECE and ECRH (EC-17), Deurne, 2012, EPJ Web of Conferences 32 (2012) 02005.

[49] W.W. Eshetu et al, Proc. $17^{\text {th }}$ Joint Workshop on ECE and ECRH (EC-17), Deurne, 2012, EPJ Web of Conferences 32 (2012) 03011. 